ISSN 1991-8631

Original Paper

http://indexmedicus.afro.who.int

\title{
Catalogue des plantes médicinales utilisées dans le traitement de la lithiase rénale dans la province de Tan-Tan (Maroc saharien)
}

\author{
Mohamed GHOURRI, Lahcen ZIDANE et Allal DOUIRA*
}

\author{
Université Ibn Tofaïl, Faculté des Sciences, Laboratoire de Botanique et de Protection des Plantes, \\ B.P. 133, Kénitra, Maroc. \\ *Auteur correspondant, E-mail : douiraallal@hotmail.com
}

\section{RESUME}

Une étude ethnobotanique a été réalisée dans la région de Tan-Tan pour établir le catalogue des plantes médicinales utilisées dans le traitement de la lithiase rénale et collecter le maximum d'informations sur les recettes thérapeutiques pratiquées par la population locale. A l'aide de 350 fiches questionnaires, des enquêtes ethnobotaniques ont été réalisées dans la région de Tan-Tan pendant quatre campagnes de terrain, entre 2007 et 2011. L'analyse des données recueillies sur le terrain a permis de recenser 50 espèces appartenant à 29 familles dont celle des Apiaceae, la plus représentée avec 7 espèces. De même, les résultats obtenus montrent que la majorité des recettes thérapeutiques utilisées dans le traitement de la lithiase sont préparées à partir des feuilles ou des graines sous forme de décoction. Les informations rapportées par cette étude, pourraient constituer une base de données pour les recherches ultérieures dans le domaine de la phytochimie dont le but d'identifier de nouveaux principes actifs naturels utilisables en pharmacologie.

(C) 2013 International Formulae Group. All rights reserved.

Mots clés : Lithiase rénale, enquête ethnobotanique, plantes médicinales, usages thérapeutiques, catalogue floristique, Tan-Tan.

\section{INTRODUCTION}

La lithiase urinaire est reconnue depuis les temps les plus reculés et s'avère indissociable de l'histoire de l'humanité. Elle touche, selon les pays, de 4 à $20 \%$ de la population avec un taux de récidive qui avoisine les $50 \%$. Plusieurs facteurs sont responsables de la formation des calculs urinaires notamment les conditions sanitaires, les habitudes alimentaires et le niveau de vie de la population (Oussama et al., 2000).

Actuellement, dans les pays industrialisés, les calculs rénaux représentent $90 \%$ des calculs urinaires qui se caractérisent par la précipitation solide de solutés à une concentration excessive dans les urines (calcium, phosphate, oxalates, acide urique...). Cependant, il existe quatre principaux types de lithiases, classées selon leur composition: les lithiases calciques, d'acide urique, de cystine et les lithiases de phosphate.

Au Maroc, les études réalisées sur la lithiase urinaire restent peu nombreuses, elles se sont intéressées à la détermination de la composition des calculs collectés dans certaines régions (Al Kabbaj, 2000 ; Oussama et al., 2000; Fekak, 2006; Laziri, 2009 ). Ces 
études ont montré la prépondérance de l'oxalate de calcium monohydrate comme constituant majoritaire des calculs urinaires.

L'analyse des calculs apporte des informations qui peuvent contribuer efficacement à la compréhension des mécanismes impliqués dans leur formation (Daudon et al., 1999). Elle devrait être en premier temps de l'exploration étiologique. L'identification des causes de la lithiase permet de lui opposer des mesures thérapeutiques et diététiques efficaces conduisant à la prévention, la réduction ou l'arrêt des récidives calculeuses.

Dans ce contexte, l'étude ethnobotanique entreprise a pour objectif d'identifier et d'inventorier les plantes médicinales utilisées dans le traitement des affections rénales tout en apportant des précisions sur la nomenclature des plantes utilisées, la partie utilisée, le mode de préparation et la maladie traitée.

\section{MATERIEL ET METHODES}

Description de la zone d'étude

La province de Tan-Tan se situe au sud-ouest du Maroc. Elle est limitée au nord par la province de Guelmim, à l'Ouest par l'océan atlantique, au sud par la province de Laâyoune, à l'est par la province d'Es-Smara et au nord-est par la province d'Assa-Zag (Figure 1). Cette région s'étend sur une superficie de $17295 \mathrm{~km}^{2}$ avec une population de 70147 habitants dont 3041 au rural (3\%) et 67105 en milieu urbain (97\%) (MP, 2007).

Les grands traits géomorphologiques de la province s'inscrivent dans de grands domaines relativement simples à embrasser : de grands plateaux d'érosion armés de croûtes calcaires résistantes partiellement couvertes de dépôts limoneux d'origine fluviale ancienne et décalés de $200 \mathrm{~m}$ en altitude. Ce sont au SE, la Hamada du Draâ (carbonifère) qui forme un immense entablement d'une grande monotonie; à l'W, les hamadas côtières plus basses et aussi plus lanières et plus déformées par des mouvements tectoniques post-villafranchiens, et un bas plateau d'une altitude moyenne d'environ 30 m (secondaires et tertiaires) emboité dans les hamadas, couverts d'une dalle très résistante ou de formations grésocalcaires dunaires, ou encore de sables mobiles. Ce dernier est défoncé par un système de sebkhas que la présence de couches salines rend quasiment abiotiques (André, 1973).

Les sols de la province de Tan-Tan sont classés soit parmi les sols marrons gris, calcaires, à régime aéropédique et à faciès «forestier », moyennement riches en humus, soit parmi les sols marrons gris et à faciès «isohumique», calcaires, à régime aéropédique, moyennement riches ou pauvres en humus (Bryssine, 1973).

Le réseau hydrographique de la province de Tan-Tan est constitué de 5 Oueds principaux : Draâ, Elwaêr, Chbika, Oum Fatma et Ben khlil. Ils sont caractérisés par des écoulements sporadiques et de courte durée (El Garmai et al., 2003).

Le territoire de la province ne contient aucune nappe phréatique mais il est intéressant de signaler la présence des nappes alluviales à fortes teneurs en salinité (El Garmai et al., 2003).

La région de Tan-Tan se trouve dans l'étage bioclimatique infra méditerranéen saharien à hiver tempéré. Elle est fortement marqué par l'influence de l'alizé maritime dans la zone côtière: forte humidité de l'air, nébulosité appréciable, températures modérées et amplitudes thermiques diurne et annuelles faibles, vents réguliers (de NNE dominant), pluies insignifiantes (Delannoy, 1973).

Ce climat fortement original par rapport à celui du Sahara continental ou côtier oriental, se dégrade progressivement vers l'intérieur (Delannoy, 1973).

L'étage bioclimatique méditerranéen saharien est un milieu écologique bien individualisé, où le jeu de compensation entre les températures journalières, saisonnières et annuelles (moyennes maximale les plus fortes en été) et les précipitations (moyennes annuelles les plus faibles), ne peut permettre (sauf dans des conditions locales bien particulières : oasis, fond d'oued) qu'un faible 
potentiel végétatif; la végétation est de type steppique, à éléments bien propres (xérophiles et thermophiles), discontinue et structurée par un xérophyte en touffes. C'est le milieu ayant la végétation la plus xérophile, présentant les plus fortes amplitudes de température, les précipitations les plus faibles et la plus longue période de sécheresse de tous les étages de la zone aride du pays (Ionesco, 1965).

La biodiversité végétale du Sahara a été prouvée par deux travaux antérieurs qui ont été abordés sur des provinces sahariennes notamment celui de Birouk (1991) qui a dressé un catalogue des plantes vasculaires renfermant 480 espèces, réparties en 65 familles botaniques ainsi que celui de Mathez et Sauvage (1974) qui ont recensé 297 plantes vasculaires dans la province de Tarfaya, incluant à l'époque la région de Tan-Tan.

La région de Tan-Tan était un poste militaire espagnol vers la fin du dernier siècle. Son véritable développement était lié à la Marche Verte et à la construction du port d'El Ouatia vers l'année 1978 (SRAT, 2010).
Dans cette région, la pêche maritime constitue une activité socio-économique primordiale du point de vue de leur rendement halieutique malgré les infrastructures de base qui sont peu développées et le système de production industrielle qui a connu dernièrement l'installation de 20 unités de transformation des poissons. En effet, le port de Tan-Tan est devenu rapidement parmi les premiers ports sardiniers du Maroc. Par conséquent, il offre un emploi pour 12.000 personnes, ce qui représente $60 \%$ de la population active. Le reste est occupé par l'Administration provinciale et le commerce au niveau local (SRAT, 2010).

\section{Méthodologie}

Ce travail a été réalisé sur la base d'enquêtes ethnobotaniques pour rassembler le maximum d'informations sur la diversité floristique et les usages thérapeutiques des plantes médicinales utilisées dans le traitement des affections rénales dans la région de Tan-Tan.

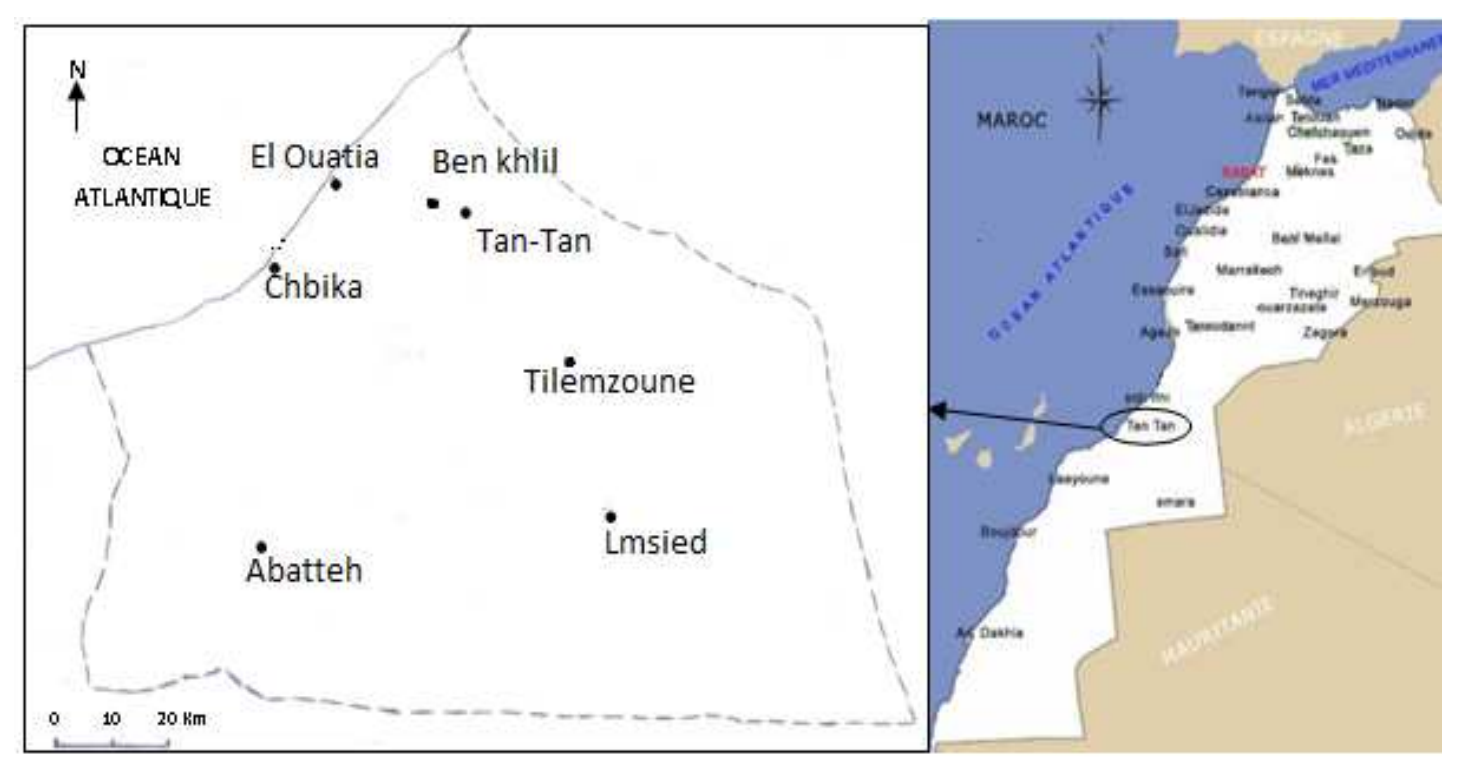

Figure : Localisation de la province de Tan-Tan. 
A travers un échantillon aléatoire de 350 personnes, la population des sept communes (El Ouatia, Chbika, Tan-Tan, Lmsied, Tilemzoune, Abatteh et Ben Khlil) de la province de Tan-Tan a été enquêtée entre 2007 et 2011.

A l'aide d'un questionnaire, les enquêtes ont permis de recueillir des informations précises sur les pratiques thérapeutiques utilisées par la population de cette région dans le traitement de la lithiase rénale (nom vernaculaire de la plante, partie utilisée et mode de préparation).

\section{RESULTATS}

Les plantes médicinales recensées au cours de cette étude ethnobotanique auprès de la population de la province de Tan-Tan sont réparties en 29 familles et 50 espèces utilisées dans le traitement de la lithiase rénale.

Le catalogue des plantes médicinales ci dessous montre les différents modes d'utilisation de ces espèces (décoction, infusion, macération, poudre,...) dans des recettes utilisées dans le traitement des affections rénales.

\section{Catalogue des plantes médicinales}

ALLIACEAE

Allium cepa $\mathrm{L}$.

Nom français: Oignon

Nom vernaculaire : L-besla

Partie utilisée : Le bulbe

Utilisation locale :

La décoction d'Allium cepa coupée associée à Petroselinum sativum (Ma'dnous), mélangée à l'huile d'olive, est recommandée contre les calculs rénaux.

Allium sativum $\mathrm{L}$.

Nom français : Ail

Nom vernaculaire : Toum

Partie utilisée : Le bulbe

Utilisation locale :

Un mélange à base de Petroselinum sativum et d'Allium sativum (Toum), en décoction, est utilisé contre les calculs rénaux.

\section{AMARANTHACEAE \\ Atriplex halimus L.}

Nom français : Arroche sauvage, Pourpier de mer ;

Nom vernaculaire : Legtef (continental)

Partie utilisée : La feuille

Utilisation locale :

Les feuilles sont utilisées, en décoction, contre les calculs rénaux à raison de $250 \mathrm{~g}$ de feuilles par litre d'eau.

\section{APIACEAE}

Ammi visnaga (L.) Lamk

Nom français : Ammi visnage

Nom vernaculaire : Bûŝnîkha

Partie utilisée : Les ombelles

Utilisation locale :

Une recette à base d'Ammi visnaga et d'une faible quantité de Crocus sativus (Za'fran lhor), en décoction à l'eau minérale (Sidi Hrazem), est employée contre la lithiase urinaire.

Ammodaucus leucotrichus Coss. \& Dur.

Nom français : Cumin velu

Nom vernaculaire : Kmoun reg

Partie utilisée : La graine

Utilisation locale :

Un remède à base d'Ammodaucus leucotrichus découpée, de la poudre de Myristica fragrans (Gouza), d'Euphorbia falcata (Hayat noufos) (1 cuillère), d'Herniaria hirsuta (harast lhjar) (1 cuillère), d'un seul fruit de citron entier et d'une tasse de l'huile d'olive, en décoction à trois litres d'eau minérale (Sidi Hrazem) jusqu'à ce qu'il devienne jaune, réduire au quart de volume initial. Le filtrat mélangé au jus de citron et à l'huile d'olive (deux tasses) sert à traiter la lithiase.

\section{Apium graveolens L.}

Nom français : Céleri

Nom vernaculaire : Krâfes

Partie utilisée : La graine et la tige feuillée

Utilisation locale :

Une décoction d'Apium graveolens associé à Eugenia caryopyllata (Qrenfel) est prise contre les calculs rénaux en raison de deux tasses par jour (matin et soir). 


\section{Daucus carotta L.}

Nom français : Carotte cultivée

Nom vernaculaire : Khizzu

Partie utilisée : La graine

Utilisation locale :

Une recette à base de graines de Daucus carotta, de graines de Midicago sativa, d'Herniaria hirsuta (Harast lhjar), de Glycirrhiza glabra ('erq souss), en décoction à l'eau minérale (Sidi Ali), est employée contre les calculs rénaux.

Eryngium triquetrum Lam.

Nom français : Panicaut à feuilles de chêne

Nom vernaculaire : Zreyga

Partie utilisée : La tige feuillée

Utilisation locale :

Une recette à base de Thymus Broussonetii, d'Herniaria hirsuta (Harast lhjar), d'Ammodaucus leucotrichus (Kamoun reg), d'Anastatica hierochuntica (Kemcha), de Rosmarinus officinalis (Lyazir), de Petroselinum sativum (Ma'dnous), d'Opunitia ficus-indica (Nowar aknari), de Zea mays (Hrir lkbal) et d'Eryngium triquetrum (Zriyga), en décoction, est administrée contre la lithiase.

\section{Foeniculum vulgare Mill.}

Nom français: Fenouil

Nom vernaculaire : Nafa', Besbasse

Partie utilisée : La graine

Utilisation locale :

L'infusion d'une petite quantité des graines par un litre d'eau bouillante, pendant trente minutes, est préconisée contre la lithiase.

Petroselinum sativum Hoffm.

Nom français : Persil

Nom vernaculaire : Ma'dnous

Partie utilisée : La tige feuillée et la graine

Utilisation locale :

La plante entière, en décoction, est employée pour combattre les calculs rénaux. Le persil associé à l'Herniaria hirsuta (Harast lhjar), en décoction, est utilisé contre les calculs rénaux.

Une décoction de Petroselinum sativum mélangée au jus de citron est très conseillée contre la lithiase.
Un mélange à base de Petroselinum sativum et d'Allium sativum (Toum), en décoction, est utilisé contre les calculs rénaux.

Une recette à base de Petroselinum sativum et d'Herniaria hirsuta (Harast lhjar), en décoction à l'eau minérale (Sidi Hrazem), est utilisée contre la lithiase.

Le décocté de Petroselinum sativum à l'eau minérale (1 verre) mélangé au miel (1 cuillère) et au jus de deux citrons, est utilisé contre la lithiase pendant une durée de quinze jours.

Un mélange à base de Petroselinum sativum (un bouquet), d'Herniaria hirsuta (Harast lhjar, poignée) et d'Euphorbia falcata (Hayat noufous, poignée), en décoction dans 1,5 litres d'eau minérale (Sidi Hrazem) jusqu'à ce qu'il devienne jaune, puis réduit jusqu'à $1 / 4 \mathrm{du}$ volume initial. Le filtrat tiède mélangé au jus d'un citron et à deux cuillères d'huile d'olive est utilisé contre la lithiase à raison de trois fois par jour.

Un mélange à base de Petroselinum sativum (un bouquet), d'Herniaria hirsuta (Harast lhjar) et de Crocus sativus (Za'fran lhor), en décoction, est employé contre la lithiase.

Un mélange à base de Petroselinum sativum (un bouquet), d'Herniaria hirsuta (Harast lhjar) et de Juncus maritimus (Zri't smar) en poudre, d'Opunitia ficus-indica (Nowar aknari), de Ziziphus lotus (Nbeg) en poudre, en décoction à l'eau minérale (Sidi Hrazem), est pris contre la lithiase.

ASTERACEAE

Bubonium graveolens (Forssk) Maire

Nom français : Astérisque maritime

Nom vernaculaire : Tâfsa

Partie utilisée : sommités fleuries

Utilisation locale :

La décoction de Bubonium graveolens est utilisée, pendant une semaine, comme un traitement de la lithiase.

\section{BRASSICACEAE}

Anastatica hierochuntica L.

Nom français : Rose de Jéricho

Nom vernaculaire : L-kemša

Partie utilisée : La tige feuillée 
Utilisation locale :

L'Anastatica hierochuntica, en décoction, est utilisée contre les calculs rénaux à raison de $250 \mathrm{~g}$ par un litre d'eau.

Lepidium sativum L.

Nom français : Cresson alenois

Nom vernaculaire : Hab Rchad

Partie utilisée : La graine

Utilisation locale :

Un mélange de graines de Lepidium sativum et de graines de Nigella sativa, moulues, mélangé au miel, est prescrit contre les calculs rénaux.

\section{BURSERACEAE}

\section{Commiphora africana}

Nom français: Bdellium africain

Nom vernaculaire : ûmm-en-nâs

Partie utilisée : La gomme

Utilisation locale :

La poudre de la gomme associée au beurre, en décoction, est recommandée contre la lithiase à raison de deux petites tasses par jour (matin et soir).

\section{CACTACEAE}

Opunitia ficus-indica Berg.

Nom français: Figuier de barbarie

Nom vernaculaire : Aknari

Partie utilisée : Les fleurs

Utilisation locale

La décoction des fleurs est prise contre les calculs rénaux. Un mélange à base d'Opunitia ficus-indica, de Cynodon dactylon (Njem), de Zea mays (Hrir lkbal), d'Herniaria hirsuta (Harast lhjar), et de Ziziphus lotus (Nbeg), en poudre associée au miel, est utilisé contre les calculs rénaux.

Les fleurs d'Opunitia ficus-indica associées au Zea mays (Hrir lkbal), à l'Herniaria hirsuta (Harast lhjar) et au Capparis spinosa (Kebar), en décoction, sont utilisées contre les calculs rénaux à raison d'une tasse par jour.

\section{CAMELLIACEAE}

Camellia thea Link

Nom français : Thé

Nom vernaculaire : Atay

Partie utilisée : Les feuilles

Utilisation locale :
L'écorce de Ziziphus lotus, en décoction avec du thé, est prescrite contre la lithiase.

\section{CAPPARIDACEAE}

Capparis spinosa $\mathrm{L}$.

Nom français : Câprier

Nom vernaculaire : Kabâr, taylilut

Partie utilisée : Le fruit

Utilisation locale :

Un mélange à base d'Opunitia ficusindica, de Cynodon dactylon (Njem), de Zea mays (Hrir lkbal), d'Herniaria hirsuta (Harast lhjar), et de Ziziphus lotus (Nbeg), en poudre associée au miel, est utilisé contre les calculs rénaux.

Les fleurs d'Opunitia ficus-indica associées au Zea mays (Hrir lkbal), à l'Herniaria hirsuta (Harast lhjar) et au Capparis spinosa (Kebar), en décoction, sont utilisées contre les calculs rénaux à raison d'une tasse par jour.

\section{CARYOPHYLLACEAE}

Herniaria hirsuta L.

Nom français : Herniaire

Nom vernaculaire : Harasst lhjar

Partie utilisée : Toutes les parties de la plante Utilisation locale :

La plante entière, en décoction dans l'eau minérale (Sidi Hrazem), est employée contre les calculs rénaux.

Les feuilles, en décoction avec du thé, sont utilisées contre les calculs rénaux à raison d'une poignée deux fois par jour.

Les feuilles d'herniaire associées à celles de Lavandula dentata (khzama), en décoction, sont utilisées contre le froid et les calculs rénaux à raison d'une cuillère de chaque plante par un litre d'eau.

Les feuilles, en poudre, sont employées contre les calculs rénaux à raison d'une cuillère par jour.

Une recette à base d'Herniaria hirsuta, de Zea mays (Hrir lkbal), de Cynodon dactylon (Jdour njem), et d'Opunitia ficusindica (Nowar aknari), en décoction dans l'eau minérale (Sidi Hrazem), est prescrite contre la lithiase rénale.

Un mélange à base d'Herniaria hirsuta, de Zea mays (Hrir lkbal), 
d'Euphorbia falcata (Hayyat nofos), d'Opunitia ficus-indica (Nowar aknari), de Nigella sativa (Sanouj) et de Lavandula dentata (Khozama), en décoction, est recommandé contre la lithiase.

La poudre d'Herniaria hirsuta associé au miel, est utilisée contre la lithiase à raison de deux cuillères par jour (matin et soir).

Un remède à base d'Herniaria hirsuta, d'Opunitia ficus-indica (Nowar aknari), de Lavandula dentata (Khozama) et d'Origanum compactum (Azokanni), en décoction avec du thé, est utilisé contre la lithiase.

Herniaria hirsuta associée à l'Euphorbia falcata (Hayyat nofos), en décoction, est utilisée contre la lithiase à raison de deux tasses par jour (matin et soir).

Une recette à base d'Herniaria hirsuta, de Zea mays (Hrir lkbal), d'Opunitia ficusindica (Nowar aknari), de Ziziphus lotus (Nbeg), de Petroselinum sativum (Ma'dnous), en décoction, mélangée au jus de citron, est administrée, le matin à jeun, contre la lithiase.

La décoction d'Octopus ajoutée au Herniaria hirsuta, en décoction, est utilisée, avant les repas, contre la lithiase à raison de trois tasses par jour.

\section{EUPHORBIACEAE}

\section{Euphorbia falcata L.}

Nom français : Euphorbe en faux

Nom vernaculaire : Hayyat en-nufûs

Partie utilisée : La plante entière

Utilisation locale :

Une préparation à base de Crocus sativus, d'Origanum comactum (Azokanni), d'un bouquet d'Euphorbia falcata (Hayyat nofos), d'un bouquet d'Herniaria hirsuta (Harast lhjar), et de Ziziphus lotus (Nbeg), en poudre portée à ébullition dans trois litres d'eau minérale jusqu'à réduction à un litre. Le volume de deux tasses de filtrat mélangé à 1,5 litre d'eau minérale (Sidi Hrazem), est utilisé, pendant trois jours, contre la lithiase.

Euphorbia granulata Forsk.

Nom français : Euphorbe

Nom vernaculaire : Kbidet ed-dobb

Partie utilisée : La racine
Utilisation locale :

L'Euphorbia granulata, en poudre, est prescrite contre la lithiase rénale.

\section{FABACEAE}

Cassia festula L.

Nom français : Caneficier, Grande casse

Nom vernaculaire : 'ûd salib, kharûb 1-hindi

Partie utilisée : Les gousses

Utilisation locale :

Un mélange à base de Cassia festula, d'Herniaria hirsuta (Harast lhjar), d'Opunitia ficus-indica (Nowar aknari), de Cynodon dactylon (Njem), en décoction, est préconisé contre la lithiase.

\section{Glycirrhiza glabra L.}

Nom français : réglisse

Nom vernaculaire : 'arq souss

Partie utilisée : La racine

Utilisation locale :

Une recette à la base de Glycirrhiza glabra, de Ziziphus lotus (Nbeg), de faible quantité de Nigella sativa (Sanouj), en décoction dans l'eau minérale, est employée contre les calculs rénaux.

\section{Medicago sativa L.}

Nom français : luzerne

Nom vernaculaire : Fessa

Partie utilisée : La graine

Utilisation locale :

Une recette à base de graines de Daucus carotta, de graines de Midicago sativa, d'Herniaria hirsuta (Harast lhjar), de Glycirrhiza glabra ('erq souss), en décoction à l'eau minérale (Sidi Ali), est employée contre les calculs rénaux.

\section{Ononis natrix L.}

Nom français : Coquecigrue

Nom vernaculaire : Hennet reg

Partie utilisée : La tige feuillée

Utilisation locale :

Les tiges feuillées d'Ononis natrix, en décoction, sont utilisées contre la lithiase.

\section{IRIDACEAE}

Crocus sativus L.

Nom français : Vrai safran

Nom vernaculaire : Za' frane lhor

Partie utilisée : Les stigmates des fleurs 
Utilisation locale :

Une préparation à base de Crocus sativus, d'Origanum comactum (Azokanni), d'un bouquet d'Euphorbia falcata (Hayyat nofos), d'un bouquet d'Herniaria hirsuta (Harast lhjar), et de Ziziphus lotus (Nbeg), en poudre portée à ébullition dans trois litres d'eau minérale jusqu'à réduction à un litre. Le volume de deux tasses de filtrat mélangé à 1,5 litre d'eau minérale (Sidi Hrazem), est utilisé, pendant trois jours, contre la lithiase.

Un mélange à base de Crocus sativus et de Ziziphus lotus (Nbeg), en décoction à l'eau minérale, mélangée au miel, est prescrit contre les calculs rénaux.

Un gramme de Crocus sativus, en décoction au miel, est utilisé pour mobiliser les calculs dans les reins à raison de deux petites cuillères par jour.

Une recette à base de Crocus sativus (1 $\mathrm{g})$, de jus de citron (une demi-tasse) et du miel $(250 \mathrm{~g})$, est conseillée contre la lithiase à raison de deux cuillères par jour (matin et soir).

\section{JUNCACEAE}

Juncus maritimus Lank.

Nom français : Jonc maritime

Nom vernaculaire : Smâr

Partie utilisée : La graine

Utilisation locale

Un mélange à base de Juncus maritimus, de Petroselinum sativum (Ma'dnous), et d'Herniaria hirsuta (Harast lhjar), en décoction, est utilisé contre les calculs rénaux.

\section{LAMIACEAE}

Origanum compactum Benth.

Nom français : Origan

Nom vernaculaire : Azukenni

Partie utilisée : La feuille

Utilisation locale :

Une préparation à base de Crocus sativus, d'Origanum comactum (Azokanni), d'un bouquet d'Euphorbia falcata (Hayyat nofos), d'un bouquet d'Herniaria hirsuta (Harast lhjar), et de Ziziphus lotus (Nbeg), en poudre portée à ébullition dans trois litres d'eau minérale jusqu'à réduction à un litre. Le volume de deux tasses de filtrat mélangé à 1,5 litre d'eau minérale (Sidi Hrazem), est utilisé, pendant trois jours, contre la lithiase.

\section{Lavandula dentata $\mathrm{L}$.}

Nom français : Lavande vrai

Nom vernaculaire : L-khozama beldiya

Partie utilisée : La tige feuillée

Utilisation locale :

Une décoction de Lavandula dentata associée à Herniaria hirsuta (Harast lhjar) est employée, le matin à jeun, contre la lithiase à raison d'une tasse par jour.

Les tiges feuillées, en décoction, sont utilisées contre la lithiase.

\section{Lavandula multifida $\mathrm{L}$.}

Nom français : Lavande à feuilles découpées

Nom vernaculaire : Khilt lkheyl, Kohayla

Partie utilisée : La tige feuillée

Utilisation locale :

La décoction de Lavandula multifida est préconisée contre les calculs rénaux.

Un mélange à base de Lavandula multifida, d'Herniaria hirsuta (Harast lhjar), de Ziziphus lotus (Nbeg), d'Opunitia ficusindica (Nowar aknari), de Zea mays (Hrir lekbal) et de Lavandula dentata (Khozama), en décoction, est utilisé contre la lithiase.

Un remède à base de Lavandula multifida, d'Herniaria hirsuta (Harast lhjar), de Ziziphus lotus (Nbeg), d'Opunitia ficusindica (Nowar aknari), de Zea mays (Hrir lekbal), de Petroselinum sativum (Ma'dnous), de Succin (Ambre jaune, miyala lhora) en poudre, de Crocus sativus (Za'fran lhor), la moitié d'une noix de Myristica fragrans (Gouza ghlida), de Ranunculus maricatus (Wden lhalouf), en décoction dans l'eau minérale (Sidi Hrazem), réduit au quart de volume initial, est utilisé, avant les repas, contre la lithiase.

Les feuilles, en décoction avec du thé, sont utilisées contre la lithiase.

Une recette à base de Lavandula multifida et d'Herniaria hirsuta (Harast lhjar), en décoction, est prescrite contre la lithiase.

Rosmarinus officinalis L.

Nom français : Romarin

Nom vernaculaire : Yazir

Partie utilisée : Les feuilles 
Utilisation locale :

Le Romarin $(1,5 \mathrm{~kg})$, en décoction dans 1,5 litre, réduit à un litre, est utilisé pour induire la désintégration des calculs rénaux.

Thymus Broussonetii Bois

Nom français : Thym du Maroc

Nom vernaculaire : Tazûkennit

Partie utilisée : La feuille

Utilisation locale :

Une recette à base de Thymus Broussonetii, d'Herniaria hirsuta (Harast lhjar), d'Ammodaucus leucotrichus (Kamoun reg), d'Anastatica hierochuntica (Kemcha), de Rosmarinus officinalis (Lyazir), de Petroselinum sativum (Ma'dnous), d'Opunitia ficus-indica (Nowar aknari), de Zea mays (Hrir lkbal) et d'Eryngium triquetrum (Zriyga), en décoction, est administrée contre la lithiase.

\section{LAURACEAE}

Cinnamomum zaylanicum

Nom français : Cannelle de ceylan

Nom vernaculaire : Qerfa

Partie utilisée : L'écorce de l'arbre sous forme de bâtonnets

Utilisation locale

La poudre de Cinnamomum zaylanicum, en faible quantité, associée au bouillon du miel et au jus de citron, est préconisée contre la lithiase à raison d'une cuillère par jour.

\section{LINACEAE}

Linum usitatissimum L.

Nom français : Lin

Nom vernaculaire : Zeri’t 1-ketan

Partie utilisée : La graine

Utilisation locale :

Un mélange à base de Linum usitatissimum (1 verre), d'Herniaria hirsuta (Harast lhjar), de Zea mays (Hrir lkbal), de Petroselinum sativum (Ma'dnous), de Crocus sativus (Za'fran lhor, $1 \mathrm{~g}$ ) et de Ziziphus lotus (Nbeg, 3 verres), en décoction dans l'eau minérale (Sidi Hrazem), est utilisé, pendant la nuit, contre la lithiase à raison d'une cuillère par jour.

MYRISTICACEAE

Myristica fragrans Houtt

Nom français : Muscadier
Nom vernaculaire : Lgouza, Bsibisa

Partie utilisée : La noix et le macis

Utilisation locale :

Un remède à base d'Ammodaucus leucotrichus découpée, de la poudre de Myristica fragrans (Gouza), d'Euphorbia falcata (Hayat noufos) (1 cuillère), d'Herniaria hirsuta (Harast lhjar) (1 cuillère), d'un seul fruit de citron entier et d'une tasse de l'huile d'olive, en décoction à trois litres d'eau minérale (Sidi Hrazem) jusqu'à ce qu'il devienne jaune, réduire au quart de volume initial. Le filtrat mélangé au jus de citron et à l'huile d'olive (deux tasses) sert à traiter la lithiase.

\section{MYRTACEAE}

Eugenia caryopyllata Thunb.

Nom français : Giroflier

Nom vernaculaire : Qronfel

Partie utilisée : Les clous (boutons floraux)

Utilisation locale

Une grande tasse de clous de girofle, en décoction dans trois litres d'eau jusqu'à ce que les clous deviennent mous, puis un bouquet d'Apium graveolens (Krafes) est ajouté, puis la décoction est prolongée. Le filtrat est utilisé contre la lithiase à raison de trois tasses par jour ( 2 tasses matin et une tasse pendant le soir).

OLEACEAE

Olea europaea L.

Nom français : Olivier

Nom vernaculaire : Zaytoune

Partie utilisée : L'huile

Utilisation locale :

L'huile d'olive est utilisée, le matin à jeun, contre les calculs rénaux.

L'huile d'olive mélangé au jus de citron est employée, le matin à jeun durant une semaine, contre la lithiase à raison d'une demi-tasse de chaque ingrédient.

\section{PALMACEAE}

Phoenix dactylifera $\mathrm{L}$.

Nom français : Palmier dattier

Nom vernaculaire : Tmer

Partie utilisée : Les fruits

Utilisation locale :

Une recette à base de Zea mays, d'Herniaria hirsuta (Harast lhjar), de pépins 
des dattes ('alf tmar), d'Opunitia ficus-indica (Nowar aknari), en décoction, est utilisée contre la lithiase.

\section{POACEAE}

Aristida pungens Desf.

Nom français : Drin

Nom vernaculaire : ssbet

Partie utilisée : La feuille

Utilisation locale :

Le décocté d'Aristida pungens est utilisé contre la lithiase.

Cynodon dactylon L. Pers.

Nom français : Chiendent

Nom vernaculaire : Njem

Partie utilisée : Le rhizome

Utilisation locale :

Une recette à base de Cynodon dactylon, d'Herniaria hirsuta (Harast lhjar), et de racines de Ziziphus lotus (Jdour sder), en décoction, est utilisée contre la lithiase.

Hordeum vulgare $\mathrm{L}$.

Nom français : Orge

Nom vernaculaire : Zra', ch'ir

Partie utilisée : Les graines

Utilisation locale :

Un remède à base d'Hordeum vulgare, d'Herniaria hirsuta (Harast lhjar) et de Petroselinum sativum (Ma'dnous), en décoction, est recommandé contre la lithiase.

La décoction de l'orge est prescrite contre la lithiase.

Zea mays L.

Nom français : Maïs

Nom vernaculaire : Dra squbi

Partie utilisée : Les stigmates

Utilisation locale :

Les stigmates de maïs, en décoction, sont préconisés contre la lithiase.

Une recette à base de Zea mays, d'Herniaria hirsuta (Harast lhjar), de pépins des dattes ('alf tmar), d'Opunitia ficus-indica (Nowar aknari), en décoction, est utilisée contre la lithiase.

Un remède à base de stigmates de Zea mays, d'Herniaria hirsuta (Harast lhjar), d'Opunitia ficus-indica (Nowar aknari), et de Crocus sativus (Za'fran lhor), en décoction, est très recommandé contre la lithiase à raison de trois tasses par jour.
Une recette à base de Zea mays, d'Herniaria hirsuta (Harast lhjar), et d'Opunitia ficus-indica (Nowar aknari), et de Ziziphus lotus (Nbeg), en décoction dans deux litres d'eau jusqu'à réduction à un litre, est utilisée contre la lithiase à raison de deux tasses par jour (matin et soir).

Les stigmates de maïs associés au Ziziphus lotus (Nbeg), en décoction, sont recommandés contre la lithiase.

\section{POLYPODIACEAE}

Adiantum capillus-veneris L.

Nom français: Capillaire de Montpellier

Nom vernaculaire : Qzibra, qezbûr 1-bir, algalba ;

Partie utilisée : La plante entière

Utilisation locale :

La poudre d'Adiantum capillus-veneris est conseillée contre les calculs rénaux.

\section{RANUNCULACEAE}

Nigella sativa $\mathrm{L}$.

Nom français : Nigelle cultivée

Nom vernaculaire : Sanouj

Partie utilisée : Les graines

Utilisation locale :

Les graines en poudre, mélangées au miel, sont utilisées contre la lithiase à raison de quatre cuillères par un verre du miel.

Lepidium sativum (1/2 verre), en infusion jusqu'à ce qu'il devienne gonflé, associée au Nigella sativa (1/4 verre) et au miel (1/2 litre), est incubé pendant trois jour et utilisée contre la lithiase à raison de deux cuillères par jour (matin et soir).

Les graines chauffées, moulues et associées au miel d'Euphorbia officinarum (Daghmous, un litre), au beurre de chèvre (une tasse) et au Lepidium sativum (une cuillère), incubée pendant trois jours, est utilisée contre la lithiase à raison de deux cuillères par jour (matin et soir).

\section{Ranunculus muricatus L.}

Nom français : Renoncule

Nom vernaculaire : wden lhalouf

Partie utilisée : La racine

Utilisation locale :

Les racines cuites à la vapeur, associée au miel, est administrée contre les calculs rénaux. 


\section{RHAMNACEAE}

Ziziphus lotus (L.) Lank

Nom français : Jujubier

Nom vernaculaire : Ssder, Nbeg

Partie utilisée: Les fruits, les feuilles, l'écorce et les racines.

Utilisation locale :

La poudre du fruit, en décoction, est employée contre les douleurs des reins.

Les fruits associés à l'Herniaria hirsuta (Harast lhjar), en décoction, sont utilisés contre les calculs rénaux.

La décoction de $2 \mathrm{~kg}$ de fruits dans deux litres d'eau, réduite à moitié, est utilisée contre les calculs rénaux.

L'écorce de Ziziphus lotus, en décoction avec du thé, est prescrite contre la lithiase.

Les fruits associés aux racines de Glycirrhiza glabra ('erq souss), aux stigmates de Zea mays (Hrir lkbal), aux fleurs d'Opunitia ficus-indica (Nowar aknari) et à l'Herniaria hirsuta (Harast lhjar), sont utilisés, en décoction à l'eau minérale, contre la lithiase.

La décoction de racines est très appréciée dans le traitement de la lithiase.

Les feuilles associées à l'écorce, en décoction, sont utilisées contre la lithiase.

\section{ROSACEAE}

\section{Prunus domestica $\mathrm{L}$.}

Nom français : Prunier

Nom vernaculaire : Berqûq

Partie utilisée : La gomme

Utilisation locale :

La gomme de Prunus domestica, en infusion, est utilisée pour induire la désintégration des calculs rénaux.

Les fruits secs sans pépins $(500 \mathrm{~g})$, en décoction dans deux litres, est utilisée comme jus contre la lithiase à raison de deux fois par jour.

\section{RUTACEAE}

Citrus limon Riss.

Nom français : Citronnier

Nom vernaculaire : L-hamed

Partie utilisée : Les fruits
Utilisation locale :

Le jus de citron ( $1 / 2$ verre) mélangé au miel ( 1 verre) et à l'huile d'olive ( 1 verre), est utilisé contre la lithiase.

La poudre des fruits de Ziziphus lotus, associée au miel et au jus de citron, est conseillée contre les calculs rénaux à raison de deux cuillères par jour.

Le jus de citron (deux fruits), associé à du miel ( $1 \frac{1}{2}$ litre), est utilisé contre la lithiase à raison de deux cuillères par jour (matin et soir).

\section{URTICACEAE}

Forskahlea tenacissima v. cossoniana (Webb.) Batt.

Nom français : Sparadrap du désert

Nom vernaculaire : Lessig

Partie utilisée : La feuille

Utilisation locale :

Un mélange à base de Forskahlea tenacissima, d'Herniaria hirsuta (Harast lhjar), de Ziziphus lotus (Nbeg), de Lavandula dentata (Khzama), de Zea mays (Hrir lkbal), en décoction, est préconisé contre la lithiase.

Urtica urens $\mathrm{L}$.

Nom français : Ortie grièche

Nom vernaculaire : L- hurriyga 1-harcha

Partie utilisée : La plante entière

Utilisation locale :

Un mélange à base d'Ortie grièche, d'Herniaria hirsuta (Harast lhjar), d'Opunitia ficus-indica (Nowar aknari), de Zea mays (Hrir lkbal), de fruit de Ziziphus lotus (Nbeg), de feuilles de Ziziphus lotus (Wraq sder), de Petroselinum sativum (Ma'dnous), en décoction, est prescrit contre la lithiase.

La décoction d'Ortie grièche est utilisée contre la lithiase à raison de deux tasses par jour (matin et soir).

\section{VITACEAE}

Vitis vivifera $\mathrm{L}$.

Nom français : Vigne

Nom vernaculaire : Zbîb

Partie utilisée : Le fruit

Utilisation locale

Les raisins secs, en décoction dans l'eau minérale (Sidi Hrazem), sont utilisés, le matin à jeun, contre les calculs rénaux. 


\section{DISCUSSION}

La population de la province de TanTan a accumulé un véritable savoir sur les vertus des plantes médicinales. Cette étude avait pour but de mieux connaitre et de valoriser les usages médicinaux liés à la flore locale utilisée dans le traitement des affections rénales dans la région de Tan-Tan.

Dans cette étude, nous avons recensé 50 espèces médicinales utilisées dans le traitement traditionnel de la lithiase rénale. En effet, ces résultats ont été confirmés par des études similaires effectuées dans d'autres régions du Maroc, citons à titre d'exemple :

- Hseini (2007) a montré que l'espèce Herniaria hirsuta à une efficacité importante dans la thérapie des calculs rénaux dans la région du Rabat, même son nom vernaculaire en arabe "Harasset lahjar" prouve son action thérapeutique. De même, d'autres espèces telles que Zea mays, Ziziphus lotus, Opuntia ficus-barbarica et Juncus sp. sont surtout prescrites comme remède aux maladies de l'appareil urinaire.

- Lahsissene (2009) a mentionné que l'espèce Opuntia ficus-indica, Herniaria glabra, Euphorbia flacata, Zea mays, Agropyrum repens, Juncus acutus, Ziziphus lotus, sont préconisées contre la lithiase rénale par contre l'espèce Linum usitatissimum est très conseillée contre les infections urinaires dans la région de Zaër (Maroc)

- Salhi et al. (2010) ont indiqué que l'espèce Ammi visnaga et Herniaria hirsuta sont utilisées contre les calculs rénaux alors que Glycyrrhiza glabra, Lavandula dentata, Zea mays, Opuntia ficus-indica, Hordeum vulgare, et Agropyrum repens sont recommandées contre les infections urinaires dans la région de Kénitra (Maroc).

En définitive, cette étude comparative montre que la flore médicinale, utilisée dans le traitement des affections rénales dans la région de Tan-Tan, est plus riche en espèces médicinales que les flores médicinales inventoriées ailleurs.

\section{Conclusion}

Les enquêtes ethnobotaniques que nous avons menées dans la province de Tan-Tan ont montré que la lithiase rénale est une pathologie très fréquente chez la population de cette région. Elle est liée aux modifications des habitudes nutritionnelles, des conditions sanitaires, des facteurs d'environnement ou de la prévalence des pathologies qui prédisposent au risque de la lithiase.

Les concepts de traitement médical de la lithiase rénale ont été précisés depuis longtemps pour stabiliser l'évolution des calculs et de prévenir les récidives. Le but ultime de cette prise en charge médicale repose sur des enquêtes étiologiques systématiques et nécessite une synthèse des données cliniques, radiologiques et biologiques basées sur l'analyse des calculs rénaux.

Dans cette région, la population utilise la phytothérapie pour diminuer les complications de la lithiase rénale à l'aide des recettes thérapeutiques constituées par des plantes médicinales anti lithiasiques.

Les résultats obtenus montrent que parmi les 29 familles recensées, comprenant 50 espèces et 47 genres, la famille des Apiaceae est la plus représentée avec un effectif de 7 espèces (soit 14\%). Le feuillage est la partie la plus utilisée de la plante alors que la décoction est le mode d'emploi le plus pratiqué par les tradipraticiens.

Ce travail peut constituer une base de données pour la valorisation des plantes médicinales utilisées contre les affections rénales à travers des études biologiques et photochimiques des plantes inventoriées.

\section{REFERENCES}

André A. 1973. Présentation géographique de la province de Tarfaya. Contribution à l'étude scientifique de la Province de Tarfaya. Cahiers de la Recherche Agronomique (Maroc), 32: 1-13.

Bryssine G. 1973. Les sols de la Province de Tarfaya. Aperçu sommaire. Contribution a l'étude scientifique de la Province de 
Tarfaya. Cahiers de la Recherche Agronomique (Maroc), 32: 61-104.

Delannoy M. 1973. Contribution à l'étude climatologique de la province de Tarfaya. Travaux de l'Institut Scientifique Chérifien, Série générale $\mathrm{N}^{\circ} 3,15-60$.

Daudon M, Troxer O, Lechevallier E, Saussine C. 2008. Epidémiologie des lithiases urinaires. Progrès en Urologie, 18: 802-814.

El Kabbaj S, Meiouet F, El Amrani A 2000. Analyse des calculs urinaires par spectrophotométrie infrarouge à propos de 218 cas au Maroc. Biol. Santé, 1: 1423.

El Garmai A, El Maghouss M, Belyazid M, Lahroud M, Ben Abdallah K. 2003. Contribution à l'élaboration d'un pacte provincial sur la gestion des ressources naturelles au niveau de la province de Tan-Tan. Thème "Ressources naturelles et transhumance". Ministère d'Equipement et du Transport, Tan-Tan, Maroc, 3-5.

Fekak H, Sahnoun A, Rabii R, El Frougui Y, Joual A, Debbagh A, Bennani S, Oussama A, Meziane A. 2006. La lithiase urinaire : étude spectrophotométrie infrarouge (à propos de 80 cas). J. Maroc Urologie, 1: 17-20.

Hseini S, Kahouadji A. 2007. Etude ethnobotanique de la flore médicinale dans la région de Rabat (Maroc occidental). Lazaroa, 28: 79-93.

Ionisco $\quad$ T. 1965. Considérations bioclimatiques et phytoécologiques sur les zones arides du Maroc. Cahiers de la Recherche Agronomique (Maroc), 19: 55 p.

Lahsissene H, Kahouadji A, Tijane M, Hseini S. 2009. Catalogue des plantes médicinales utilisées dans la région de
Zaër (Maroc occidental). Lejeunia, 186 : $1-26$.

Laziri F, Rhazi Filali F, Oussama A, Soulaymani A, Qarro A, Lezrak M. 2010. Facteurs impliqués dans l'épidémiologie des calculs urinaires marocains. J. Maroc Urologie, 19: 9-14.

MathezA J, Sauvage C. 1975. Catalogue des végétaux vasculaires de la province de Tarfaya, in «Contribution à l'étude scientifique de la province de Tarfaya ». Trav. Insc. Sci. Chér. et Fac. Sci., Rabat, $\mathbf{N}^{\circ} 3,117-196$.

MP (Monographie Provinciale). 2007. Monographie de la province de Tan-Tan, Ministère de l'intérieur, Maroc.

Oussama A, Kzaiber F, Mernari B, Hilimi A, Semmoud A, et Daudon M. 2000. Analyse des calculs urinaires de l'adulte dans le Moyen Atlas marocain par spectrophotométrie infrarouge à transformée de fourier. Prog Urol., 10 : 404-410.

Salhi S, Fadli M, Zidane L, Douira A. 2010. Etude floristique et ethnobotanique des plantes médicinales de la ville de Kénitra, Maroc. Lazaroa, 31: 133-146.

SRAT. 2010. Etude des schémas régionaux d'aménagement du territoire des provinces du sud: Schéma régional d'aménagement du territoire de la région de Guelmim-Es Smara. Identification des espaces projets, Juillet 2010. Les bureaux d'étude URBAPLAN/CID/ANALYTICA INTERNATIONAL. Le Ministère de l'Habitat, de l'Urbanisme et de la Politique de la Ville, Guelmim, Maroc, 1204. 